\title{
Last row effect: ¿influye el sitting en los resultados del estudiante?
}

\author{
Mónica Maldonado ${ }^{a}$, Víctor Sotomayor ${ }^{\mathrm{a}}$ y Jorge Villagrasa ${ }^{\mathrm{a}}$ \\ ${ }^{a}$ Centro Universitario EDEM-Escuela de Empresarios, Valencia. \\ mmaldonado@edem.es, vsotomayor@edem.es, jvillagrasa@edem.es
}

\begin{abstract}
In the literature, several authors have analyzed how the location of the students in the classroom affects their academic performance and motivation during classes. In this sense, it should be noted that close relationships have been detected between the number of row occupied and the obtained output. This work contributes to this line of research. More specifically, it carries out a study of the aforementioned characteristics with the students of the first year of the Degree in Business Administration for Entrepreneurs at the University Center EDEM-School of Entrepreneurs of Valencia, analyzing its relative marks on attitude in class and final exam, over three subjects with totally different characters: Introduction to Business Management Studies, Introduction to Economics and Mathematics I. In particular, the results obtained allow to better understand the location of certain types of students in the classroom, being able to help the professor (and/or the educational institution) to consider their specific needs or modify their location in the classroom, in order to improve their academic performance.
\end{abstract}

Keywords: last row effect, evaluation, location, star-student

\section{Resumen}

En la literatura, diversos autores han analizado cómo la ubicación de los estudiantes en el aula afecta a su rendimiento académico y motivación durante las clases. En esta línea, cabe destacar que se han detectado estrechas relaciones entre el número de fila ocupada y el rendimiento obtenido. El presente trabajo contribuye a esta línea de investigación. Más concretamente, realiza un estudio de las citadas características con los estudiantes del primer curso del Grado en ADE para Emprendedores del Centro Universitario EDEM-Escuela de Empresarios de Valencia, analizando su nota relativa a la actitud en clase y el examen final, a lo largo de tres asignaturas con caracteres totalmente distintos: Incorporación a los Estudios de ADE, Introducción a la Economía y Matemáticas I. En particular, los resultados obtenidos permiten conocer mejor la localización de determinados tipos de estudiantes en el aula, pudiendo ayudar al docente (y/o institución educativa) a considerar sus necesidades especificas o modificar la ubicación de los mismos en el aula, con el fin de mejorar sus rendimientos académicos.

Palabras clave: efecto last row, evaluación, ubicación, star-student 


\section{Introducción}

En numerosas ocasiones, los estudiantes con mejor expediente suelen estar ubicados en las primeras filas del aula, mientras que los que obtienen peores resultados suelen hacerlo en los últimos asientos y en los que más alejados se encuentran del centro. Pero, ¿existe alguna razón para determinar por qué suele darse esta configuración?

En la literatura, la explicación más frecuente se ha centrado en argumentar que los estudiantes más inteligentes, participativos y con mayor motivación por sacar buenas notas preferirían los primeros puestos. Mientras tanto, los del fondo (y los laterales) serían más despistados, menos participativos y contarían con una motivación mucho menor. Es decir, el lugar elegido por los estudiantes, estaría muy correlacionado con su potencial, personalidad e interés (Benedict y Hoag 2004; Holliman y Anderson 1986; Pedersen 1994). A modo de ejemplo, un estudio de Smith et al. (2018) señala a los estudiantes sentados en las primeras filas y en el centro de las mismas como aquellos que muestran una mayor participación e interacción con el profesor.

Figura 1. Niveles de participación de los estudiantes

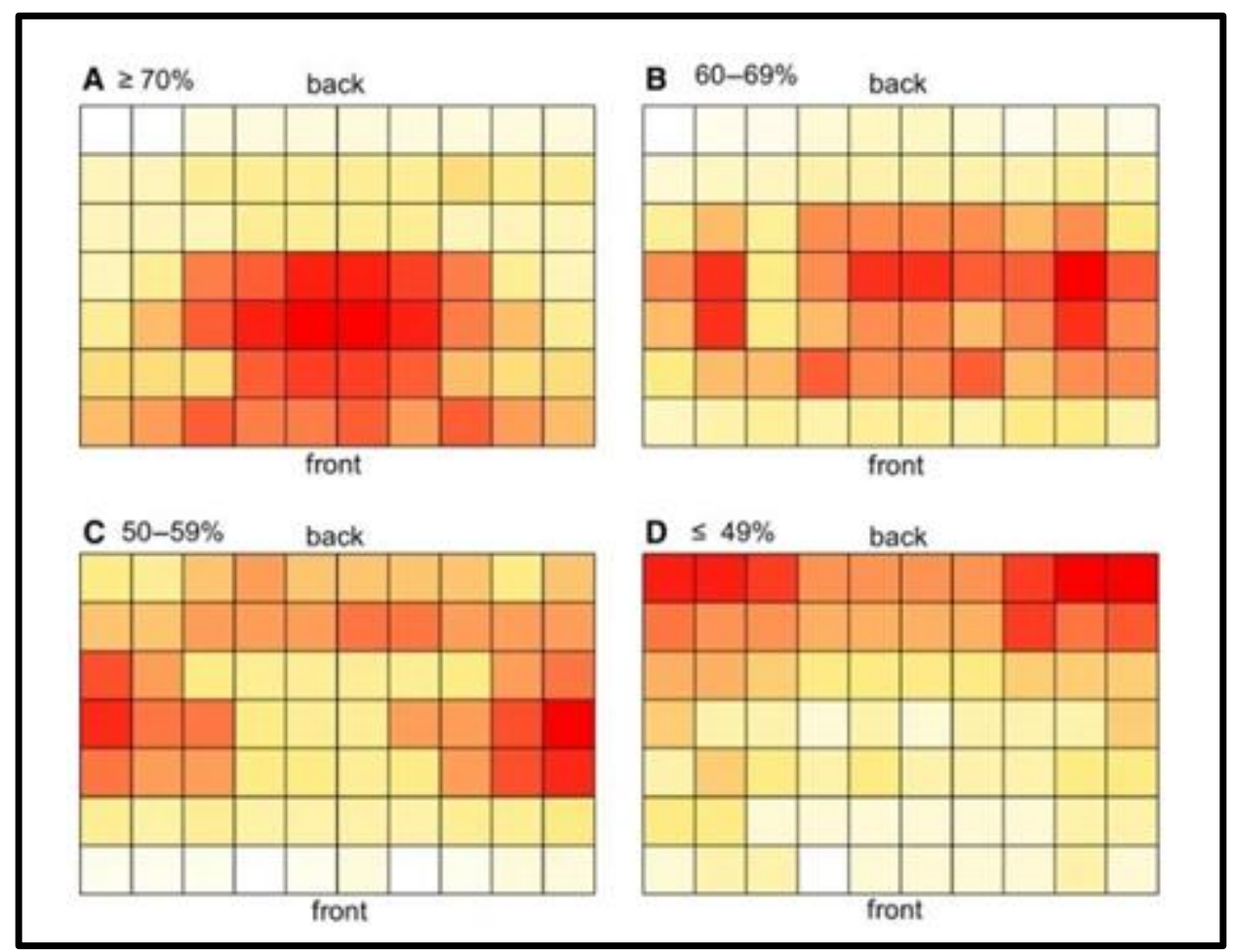

Fuente: Smith et al. (2018)

En este sentido, varias investigaciones muestran que los alumnos de las primeras filas tienden a mostrar una mayor autoestima, percibiéndose como más inteligentes y con una mejor relación con el profesor que el resto de estudiantes (Burda y Brooks, 1996). Esto, a su vez, se traduciría, en una mayor motivación, logrando de esta manera alcanzar mejores resultados (Benedict y Hoag, 2004; Holliman y Anderson, 1986).

Sin embargo, con el tiempo, numerosos autores evidenciaron que la ubicación en el aula de los estudiantes era la que verdaderamente influía en su desempeño, con independencia de sus características intrínsecas. Es decir, se demostró que la relación era justo la inversa, y que la localización en el aula no era para nada neutral (Stires, 1980). En otras palabras, se establecía que el fenómeno de que los buenos estudiantes se 
encontrasen en las primeras filas, ocurría tanto si los propios estudiantes escogían sus asientos, como si, por el contrario, se les asignaba su localización de manera totalmente aleatoria. En este sentido, la mayor atención recibida por parte de los profesores, tanto implícita como explícita, afectaría a su motivación, incidiendo en su esfuerzo por obtener unos mejores resultados académicos (Stires, 1980).

Durante algunos años, diferentes estudios arrojaron resultados contradictorios, apoyando ambas teorías y justificando sus resultados gracias a la consideración de variables como el tipo de asignatura impartida, importancia de la misma, anchura del aula, distancia de las filas al profesor, número de alumnos, etc. (Kalinowski y Toper, 2007). No obstante, la realización de experimentos mucho más sólidos y longitudinales, como el presentado por Marshall y Lonsoczy (2010) en el que se analizaba a más de 70 clases durante 15 años consecutivos, permitieron confirmar la segunda corriente de hipótesis; considerando de esta forma, a la localización en el aula, como el elemento más importante a la hora de generar mejores niveles de participación y resultados. Es decir, rechazando la hipótesis de que la ubicación de los 'listos' en las primeras filas y en el centro de las mismas fueran las responsables de unas mejores notas, y abogando porque el mero hecho de sentarse ahí hiciera a los estudiantes (independientemente de sus características) obtener mejores outputs.

El presente trabajo puede considerarse como una contribución a esta línea de investigación. De esta manera, a través del mismo, se muestran los resultados de un análisis realizado a las calificaciones de los estudiantes del primer curso académico del Grado en ADE para Emprendedores del Centro Universitario EDEM ${ }^{1}$ de Valencia. Concretamente, se estudian en detalle sus resultados académicos en tres asignaturas de distintos caracteres, con el fin de hallar una posible relación existente entre la ubicación en el aula de los alumnos y sus calificaciones obtenidas. Así, y bajo la configuración específica de las aulas de EDEM (con un número reducido de estudiantes y organizadas en forma de hemiciclo), se pretende usar esta información de cara a próximos semestres, e incluso, cursos, a la hora de poder modificar la ubicación de ciertos alumnos y aprovechar el 'efecto de la primera fila', tanto en su motivación como interés, durante cada una de las sesiones.

\section{Objetivos}

El efecto de la ubicación en el aula sobre los resultados mostrados por los estudiantes ofrece una gran oportunidad a la hora de gestionar mejor la ubicación de los mismos, ya que un conocimiento avanzado en este sentido permitiría al docente (y/o institución educativa) llegar a saber dónde se sitúan los alumnos que más ayuda necesitan, o pudiendo incluso recomendarles (o forzarles a) realizar un cambio de posición para que pudiesen aprovechar los beneficios de sentarse en los primeros puestos.

Este hecho podría además actuar como una gran oportunidad para los alumnos, los cuales podrían intentar convencer a su grupo más cercano para cambiar su ubicación y sentarse, por ejemplo, en el centro de las primeras filas, en vez de al fondo a la izquierda. De esta manera, conocer el efecto de su localización en el aula, podría automotivarles a mejorar su atención y cambiar su ubicación dentro de la misma, con el objetivo de mejorar sus calificaciones.

\footnotetext{
1 EDEM-Escuela de Empresarios, centro adscrito a la Universitat de València y a la Universitat Politècnica de València, es una institución académica fundada en 2002 bajo los auspicios de la Asociación Empresarial Valenciana (AVE) y con la premisa principal de proporcionar al sector empresarial de la región valenciana una escuela de negocios promovida y dirigida por y para las empresas. Actualmente, la institución cuenta con una oferta educativa de 43 programas, que incluyen tanto formación preuniversitaria, universitaria, como de alta dirección. Asimismo, EDEM tiene un total de 2,200 estudiantes y cuenta con la colaboración de más de 500 profesores en sus aulas.
} 
En aras de profundizar en este contexto, la presente contribución analiza el rendimiento académico obtenido por 89 estudiantes universitarios del primer curso académico del Grado en ADE para Emprendedores impartido en el Centro Universitario EDEM de Valencia. Para ello, se han utilizado algunos datos académicos de estos estudiantes generados a lo largo de tres asignaturas distintas donde los autores de este trabajo imparten docencia. De esta manera, tal y como se ha comentado en la Introducción, el principal objetivo es utilizar esta información de cara a próximos semestres (e incluso a próximos cursos) para poder modificar la ubicación de ciertos alumnos y aprovechar el 'efecto de la primera fila'. La idea principal, por tanto, sería tratar de mejorar de antemano la actitud en clase, la motivación y las calificaciones de los estudiantes que peor nivel académico presenten en un determinado momento del grado o, incluso, al inicio del mismo.

\section{Desarrollo de la innovación}

El análisis descrito en este trabajo se ha realizado utilizando diversos datos de los estudiantes de los dos grupos de primer curso del Grado en ADE para Emprendedores impartido en el Centro Universitario EDEM de Valencia. Para el estudio se han elegido tres de las asignaturas correspondientes al primer semestre de primer curso del Plan de Estudios: 'Incorporación a los estudios de ADE', 'Introducción a la Economía' y 'Matemáticas I'. Cabe mencionar que la elección de estas asignaturas se ha realizado por la consideración de las mismas como representantes adecuadas y óptimas de las tres principales áreas de conocimiento del grado: aspectos internos-entorno emprendedor (AI), aspectos externos-entorno empresarial (AE) y aspectos técnicos-herramientas de implementación (AT), respectivamente. Así, por poner un ejemplo, el resto de asignaturas impartidas durante el primer curso académico se podrían englobar perfectamente dentro de estos tres grandes bloques: 'Fundamentos de la Dirección de Empresas' y 'Derecho Mercantil', dentro de AI; 'Historia Económica y de la Empresa' y 'Microeconomía', dentro de AE; y 'Contabilidad financiera', 'Estadística Básica' y ‘Matemáticas II', dentro de AT.

Tras eliminar de la base a aquellos estudiantes que no estaban matriculados en las tres asignaturas con el objetivo de homogeneizar los resultados, el número total de estudiantes para el análisis es de 89; de los cuales, un $73 \%$ son hombres y un $27 \%$ mujeres. Las variables de las que se disponen datos son: nota de actitud y nota del examen final de las tres asignaturas, forma de acceso y nota de acceso a la Universidad, modalidad de bachillerato y tipo de centro educativo del que provienen los estudiantes, fila y posición en la que están sentados habitualmente en el aula y sexo.

Cabe mencionar que, de acuerdo con el sistema de evaluación previsto en EDEM para las asignaturas de grado, la nota final se determina a través de una ponderación simple entre la nota de evaluación continua del alumno (40\%) y la del examen final (60\%). A su vez, un $25 \%$ de la evaluación continua se obtiene a través de la nota de actitud del estudiante. Por tanto, la nota de actitud y la nota del examen final representan un $10 \%$ y un $60 \%$ respectivamente de la nota final. Son estos dos ítems de evaluación los que finalmente se han considerado para analizar los resultados académicos de los estudiantes y su posible relación con la ubicación presentada en el aula. En este caso, la nota de actitud refleja en gran medida la percepción del profesor sobre la motivación, participación, comportamiento y predisposición individual del estudiante al aprendizaje ${ }^{2}$. Por otra parte, la nota del examen final trata de reflejar los conocimientos adquiridos por los estudiantes al finalizar la asignatura.

\footnotetext{
${ }^{2}$ Es importante destacar que en este análisis no se ha utilizado la nota de evaluación continua por contener esta, aspectos más grupales (como pudieran ser las notas obtenidas por trabajos o presentaciones en grupo), y menos individuales, que desvirtuarían el objetivo específico de este trabajo.
} 
En cuanto a la nota de acceso a la Universidad se han considerado (dependiendo de la forma de acceso), la nota de las PAU, en el caso de acceso desde el bachillerato, la nota de acceso certificada por UNED, en el caso de acceso desde sistemas educativos internacionales, y la nota media del ciclo, en el caso de estudiantes que han accedido desde Ciclos formativos de grado superior. Para los estudiantes que acceden desde estudios de bachiller, también se ha considerado si han cursado la modalidad de Ciencias o de Ciencias Sociales. Asimismo, se ha tenido en cuenta la procedencia de los estudiantes, clasificándolos en centros públicos, privados o concertados.

Por último, se ha establecido la fila y la posición de los alumnos en el aula. Para ello, cada profesor situó a los alumnos según estos se sentaban en sus clases ${ }^{3}$. Posteriormente, se puso en común con el objetivo de establecer una localización única para las tres asignaturas $\mathrm{y}$, de esta forma, poder determinar si existían discrepancias significativas con algún estudiante en alguna de las clases. Tras la puesta en común se observó que no se produjeron diferencias importantes entre las tres asignaturas y que la localización del alumno se mantenía constante a lo largo del semestre; asignando, de este modo, una ubicación única para cada uno de los estudiantes. Para ello, se tuvo en cuenta la distribución del aula en la que se impartían las asignaturas (Figura 2). Esta aula, que siempre era la misma, estaba dividida en cuatro filas (siendo la primera la más cercana al profesor) y en tres posiciones (izquierda, centro y derecha), asignando de esta forma a cada estudiante una fila y una posición.

Figura 2. Distribución del aula y asignación de posición

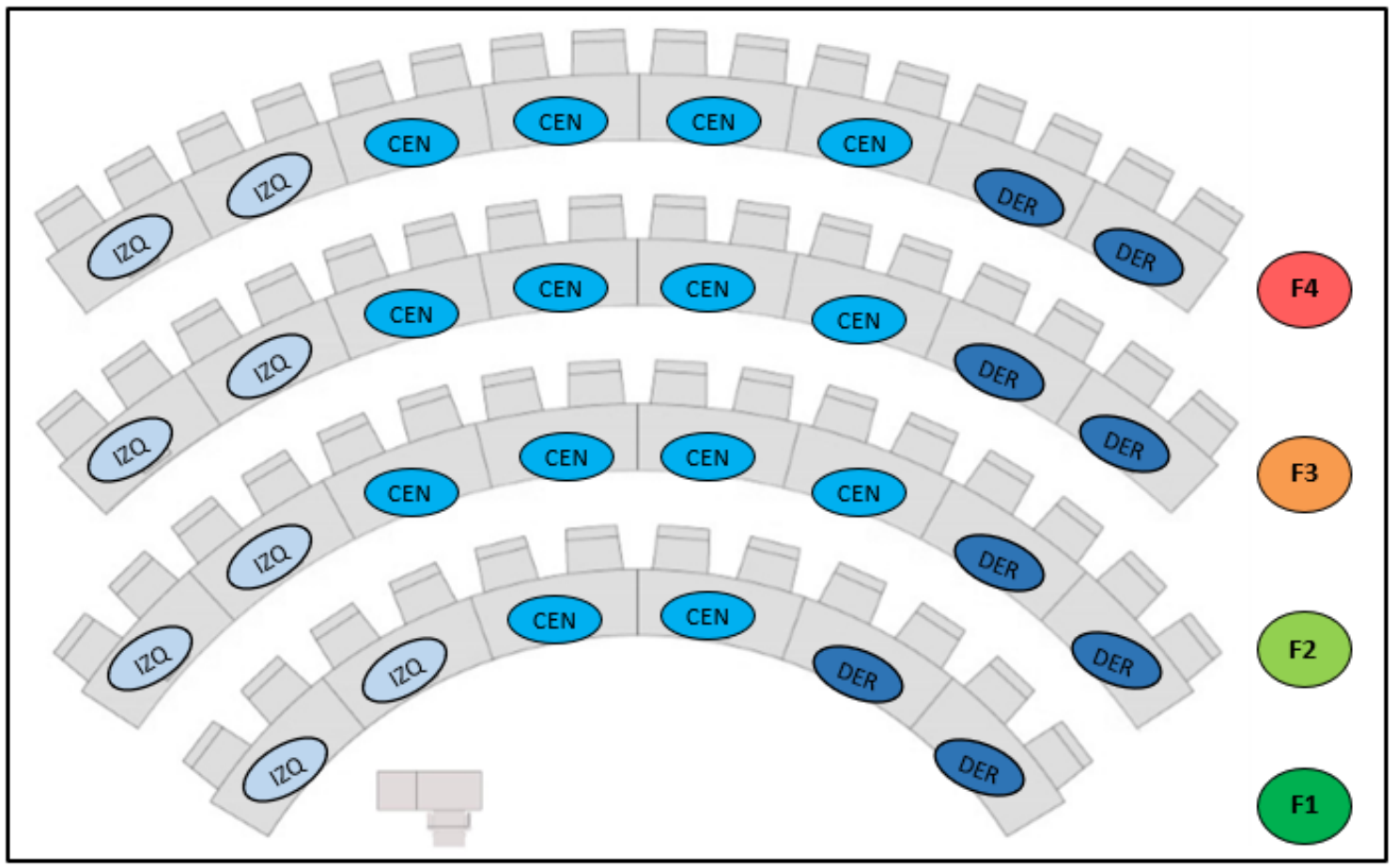

Fuente: Elaboración propia

\footnotetext{
${ }^{3}$ Nótese que los alumnos, el primer día de clase, tuvieron que sentarse forzosamente por orden alfabético, eliminándose tal restricción a partir de la segunda sesión, permitiendo de este modo al estudiante ubicarse según sus preferencias y afinidades. Sin embargo, muchos de ellos, y en concreto un tercio de los mismos, se mantuvieron en su misma fila.
} 
De las variables utilizadas, únicamente las calificaciones (actitud y examen final) y la nota de acceso son variables cuantitativas. Es por ello que, a continuación, en la Tabla 1, se presentan los valores estadísticos básicos para el conjunto de los estudiantes y para cada una de las asignaturas.

Tabla 1. Estadísticos básicos variables cuantitativas

\begin{tabular}{lccccccc}
\hline & $\begin{array}{l}\text { Introducción a la Economía } \\
\text { (AE) }\end{array}$ & \multicolumn{2}{c}{$\begin{array}{c}\text { Matemáticas } \\
\text { (AT) }\end{array}$} & \multicolumn{2}{c}{$\begin{array}{c}\text { Incorporación Estudios ADE } \\
\text { (AI) }\end{array}$} & $\begin{array}{c}\text { Nota de } \\
\text { acceso }\end{array}$ \\
\hline & Actitud & Examen & Actitud & Examen & Actitud & Examen & Global \\
\hline Mediana & 7,00 & 6,50 & 7,00 & 4,40 & 6,68 & 5,21 & 6,86 \\
Media & 7,51 & 6,42 & 7,10 & 4,42 & 6,71 & 5,16 & 7,01 \\
Desv. típica & 1,81 & 2,00 & 2,14 & 2,46 & 1,10 & 1,47 & 1,09 \\
\hline
\end{tabular}

Fuente: Elaboración propia

El resto de variables utilizadas en el análisis son cualitativas. Los valores asignados a dichas variables se muestran en la Tabla 2.

Tabla 2. Valores asignados a las variables cualitativas

\begin{tabular}{|c|c|c|c|}
\hline Variable & Nombre & Valor & Definición \\
\hline \multirow{3}{*}{ Centro de procedencia } & \multirow{3}{*}{ centro } & 1 & Público \\
\hline & & 2 & Privado \\
\hline & & 3 & Concertado \\
\hline \multirow{2}{*}{ Modalidad de Bachiller } & \multirow{2}{*}{ mod.bach } & 1 & Ciencias Sociales \\
\hline & & 2 & Ciencias \\
\hline \multirow{3}{*}{ Forma de Acceso } & \multirow{3}{*}{ acceso } & 1 & Nacional \\
\hline & & 2 & Internacional \\
\hline & & 3 & Otros \\
\hline \multirow{4}{*}{$\begin{array}{c}\text { Fila } \\
\text { (de más cercana a más alejada } \\
\text { del profesor) }\end{array}$} & \multirow{4}{*}{ fila } & 1 & Primera \\
\hline & & 2 & Segunda \\
\hline & & 3 & Tercera \\
\hline & & 4 & Cuarta \\
\hline \multirow{3}{*}{$\begin{array}{c}\text { Posición } \\
\text { (de izquierda a derecha } \\
\text { mirando de frente al aula) }\end{array}$} & \multirow{3}{*}{ posición } & 1 & Izquierda \\
\hline & & 2 & Centro \\
\hline & & 3 & Derecha \\
\hline \multirow{2}{*}{ Sexo } & \multirow{2}{*}{ sexo } & 1 & Hombre \\
\hline & & 2 & Mujer \\
\hline
\end{tabular}

Fuente: Elaboración propia

\section{Resultados}

Los resultados de esta investigación se muestran a través de la Figura 3, donde se recogen las notas de los estudiantes para cada una de las tres variables cuantitativas utilizadas en este estudio (actitud, examen final y nota de acceso), así como para la variable cualitativa sexo. Es importante subrayar que los resultados se distinguen por medias tanto de fila (fila 1, fila 2, fila 3 y fila 4) como de posición (izquierda, centro y derecha), mostrando también una media del conjunto de todos los alumnos con el objetivo de facilitar la comparación entre dichos promedios. Además, cabe destacar que con el fin de obtener resultados globales 
que pudieran afectar a decisiones a nivel de centro (y no tanto a nivel de una asignatura en particular), este trabajo utiliza datos agrupados de las tres asignaturas analizadas: 'Incorporación a los estudios de ADE', 'Introducción a la Economía' y 'Matemáticas I'.

Figura 3. Nota media de los estudiantes

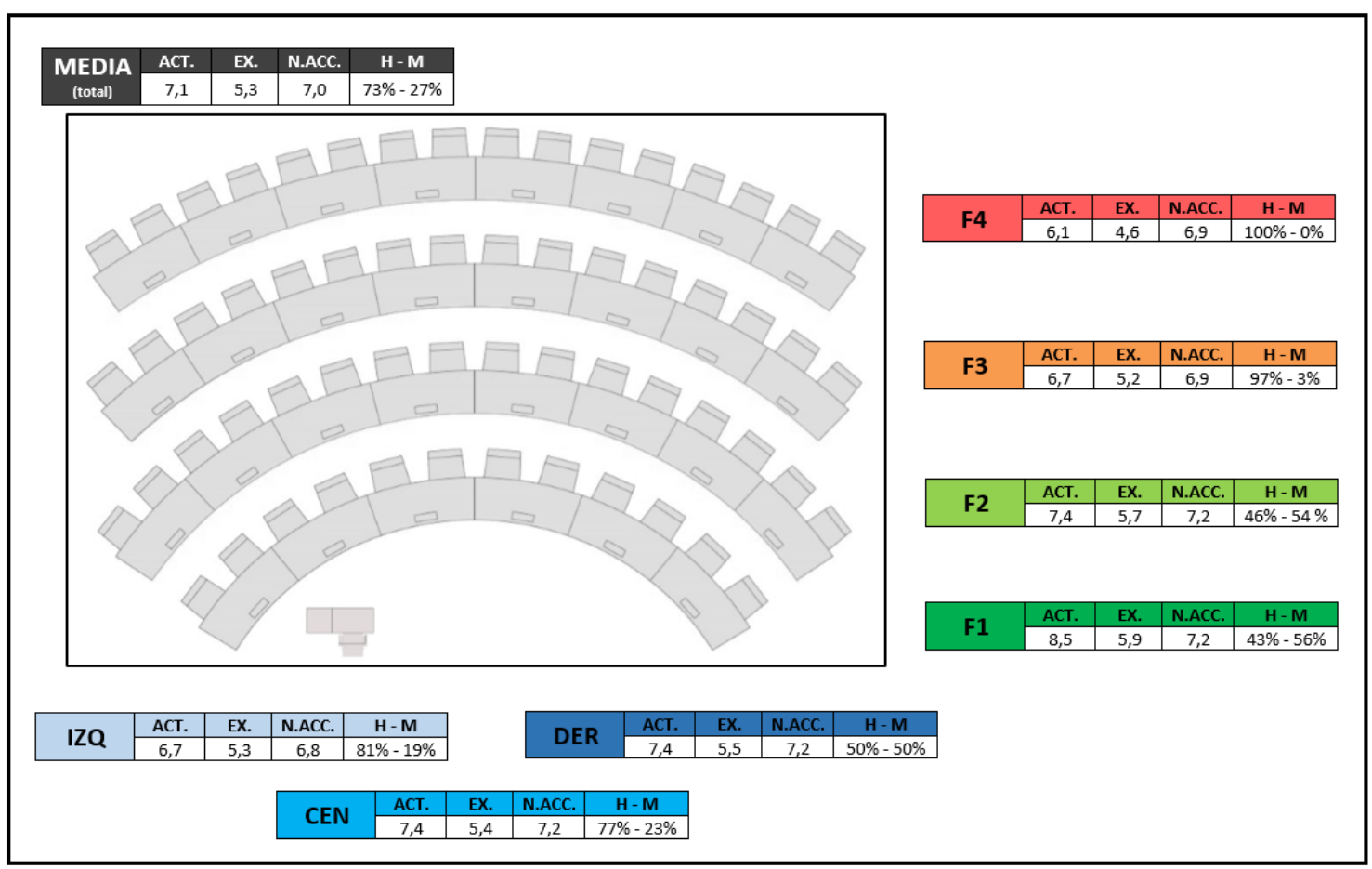

Fuente: Elaboración propia

El análisis muestra claramente cómo las notas son mucho más elevadas cuanto más hacia delante se sitúa el estudiante (con una diferencia de 2.4 puntos en actitud y 1.3 puntos en el examen final comparando la primera fila y la última) y cuanto más al centro y a la derecha se posicione (con una diferencia de 0.7 puntos en el caso de la actitud y una diferencia apenas inexistente en el caso del examen final). Además, es importante señalar que estos resultados no se verían afectados por sus notas de acceso, pues esta nota presenta valores muy parecidos en todos los casos de estudio (oscilando entre 6.8 y 7.2 en todos los casos). Es decir, los resultados mostrados en esta investigación irían en la línea del 'efecto ubicación' o 'efecto fila' defendido por la literatura a la hora de determinar por qué un estudiante obtiene un mejor rendimiento en el aula (Benedict y Hoag, 2004; Burda y Brooks, 1996; Smith et al., 2018). En otras palabras, no se estaría produciendo que los estudiantes más aventajados se sentaran en las primeras filas, sino que el simple hecho de ubicarse en esas localizaciones, les estaría ayudando a obtener mejores calificaciones. Es lo que, en este trabajo, se denomina como "evitar" el efecto pernicioso del 'last row' o 'última fila'.

No obstante, es importante recordar que según establece la literatura, para poder comprobar este tipo de efecto, los estudiantes no deberían de poder elegir su ubicación en el aula libremente. Con ello, se conseguiría evitar que únicamente los buenos estudiantes se sentaran en las primeras filas (Stires, 1980). En el caso del presente estudio, los alumnos fueron ubicados forzosamente por orden alfabético durante la primera sesión, eliminándose dicha restricción a partir de la segunda sesión y hasta final del curso. Esta flexibilidad en el criterio se realizó con el objetivo de favorecer el correcto desarrollo cognitivo y psicológico de muchos de ellos, pues según diversos estudios sobreexponer, invisibilizar o separar a un estudiante de su grupo de más cercano, podría afectar negativamente tanto a su evolución académica como 
personal (Marshall y Lonsoczy, 2010; Smith et al., 2018). Sin embargo, alrededor de un tercio de los estudiantes, se mantuvieron en sus filas, evitando así una polarización de los mejores estudiantes en estas zonas; lo cual, puede observarse perfectamente a través de los valores tan similares obtenidos respecto a la variable nota de acceso (ver Figura 3).

Asimismo, los resultados obtenidos indican que este 'efecto ubicación' en el caso particular de EDEM (y a diferencia de lo que explica la literatura de manera general) estaría ligeramente sesgado a la derecha. Es decir, los estudiantes sentados en las filas de la derecha, también obtendrían mejores resultados que el resto, algo que parece no ocurrir en estudios previos (Marshall y Lonsoczy, 2010; Smith et al., 2018). Una posible explicación podría ser el lugar específico donde se sitúa el atril en estas aulas: en la parte izquierda de la misma (ver Figura 3). Esto conllevaría que el profesor, de forma inconsciente tendiera a prestar más atención al centro o centro-derecha del aula durante sus explicaciones; un comportamiento ratificado por los tres autores de este artículo (y profesores de las asignaturas), y que podría ser el causante de dicho output.

Otro comportamiento no destacado por la literatura y que subyace de este estudio, es la localización de las mujeres en dicha muestra. Sorprendentemente, estas suelen situarse a lo largo de las dos primeras filas (con porcentajes respecto a los hombres de $56 \%$ y $54 \%$ respectivamente) y en la parte derecha del aula (con porcentajes respecto a los hombres del 50\%). Curiosamente, zonas donde mejores resultados se obtienen. De esta manera, y para mejorar la comprensión del asunto tratado, se llevó a cabo una comparación de las notas medias obtenidas por las mujeres y los hombres en sus calificaciones de actitud y examen final. El resultado fue una diferencia positiva para las mujeres de 0.8 puntos en actitud (7.7 frente a 6.9) y 0.4 puntos en examen final (5.6 frente a 5.2). Por tanto, se podría argumentar que la localización de las mujeres en las primeras filas y en la derecha, sería en parte, una posible causa de los resultados obtenidos. De hecho, tal y como se ha observado previamente, la nota de acceso es muy parecida entre filas y posiciones, por lo que la variable sexo, ganaría adeptos a la hora de ser considerada como una nueva variable relevante para explicar los resultados académicos en el aula.

Para complementar el análisis, además, se llevó a cabo un estudio de los mejores estudiantes: los llamados star-students. Es decir, aquellos que presentaban las mejores notas tanto en actitud como en examen final. Se escogieron los diez mejores alumnos para cada variable y se representaron en un gráfico idéntico al de la Figura 3 a través de estrellas: con un color morado para representar la actitud y uno amarillo para el examen final. Los resultados pueden observarse en la Figura 4, la cuál añade también una media de las calificaciones obtenidas por el conjunto de estos star-students, tal y como se hacía en la Figura 3 para el conjunto global de los estudiantes.

Los resultados en sí, no son sorpresivos, sin embargo, es la interpretación de los mismos la que hace de este análisis una herramienta muy potente e interesante para tener en cuenta en un futuro. De esta forma, se observa claramente como los mejores estudiantes, tanto en actitud como en examen final, se sentaron en las primeras filas y en el centro-derecha del aula. Tiene lógica, pues esto haría a su vez, que la media de estas áreas, fuera más elevada. En concreto, estos alumnos presentaban 1.9 puntos de media más en actitud (9.0 frente a 7.1) y 2.4 puntos más en examen final (7.7 frente a 5.3) que la media global. Pero, ¿era también porque había más mujeres que hombres entre los mejores estudiantes? ¿sacaban también las mejores estudiantes mujeres notas más altas que los mejores estudiantes hombres?

Curiosamente la respuesta a ambas preguntas fue que no. No había más mujeres que hombres entre los starstudents, sino que seguía el patrón general de toda la muestra: alrededor del $75 \%$ hombres y $25 \%$ mujeres (algo que puede observarse fácilmente analizando el borde de las estrellas, representando este con un color azul a los hombres y con un color rosa a las mujeres). Asimismo, estas mujeres star, tampoco obtuvieron 
mejores notas que los hombres, superando estas en tan solo 0.1 puntos a los hombres en actitud (9.4 frente a 9.3) y examen final (8.2 frente a 8.1).

Es por ello, que aparentemente se podría afirmar que entre los star-students también se produciría el efecto 'last row' o 'efecto ubicación' pues todos ellos se situaban en las primeras filas; aunque, sin embargo, el efecto del género no sería relevante entre los mismos, a diferencia de lo que ocurría para el conjunto de los estudiantes. Por tanto, la pregunta que faltaría por resolver sería: ¿y entre los star-students, tampoco afectaría la nota de acceso tal y como ocurría al estudiar la totalidad de los estudiantes? Curiosamente sí. Los star-students obtienen de media 0.9 puntos más de nota de acceso que el conjunto global de alumnos (7.9 frente a 7.0). Es por ello que la aportación de este trabajo se considera relevante, al establecer que el buen estudiante, en este caso el estudiante que obtiene buenas notas en bachillerato, preferiría situarse generalmente en las primeras filas. Es decir, tal y como argumentaban Benedict y Hoag (2004) o Holliman y Anderson (1986) entre otros, el lugar elegido por los estudiantes, estaría correlacionado con su potencial, personalidad e interés. O, al menos, en el caso de los mejores estudiantes. Consecuentemente, a través de esta investigación se permitiría combinar ambas teorías, aparentemente enfrentadas, bajo el estudio de diferentes tipos de estudiantes dentro de una misma aula.

Figura 4. Nota media de los estudiantes y los star-students

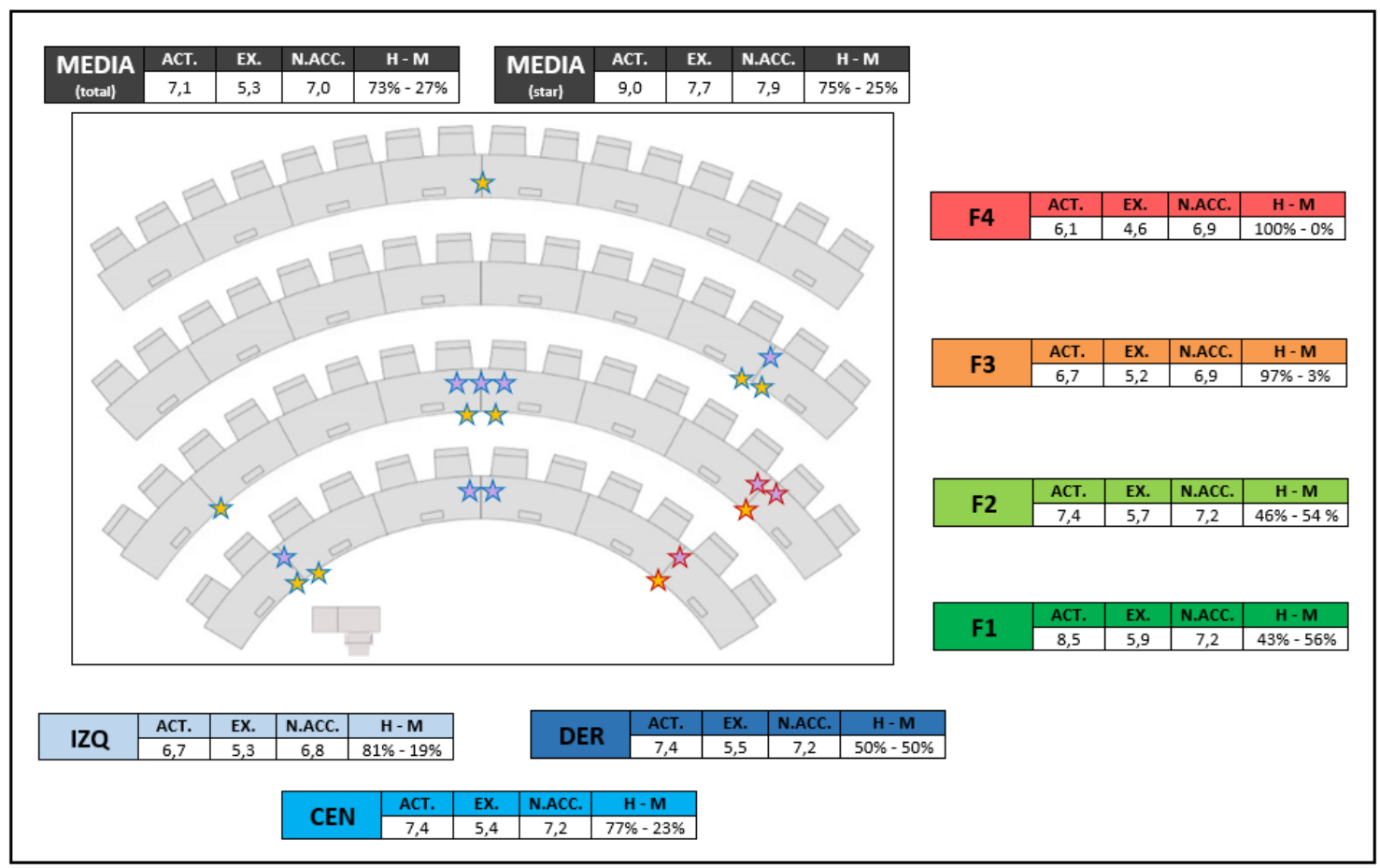

Fuente: Elaboración propia

Con el objetivo de obtener resultados más robustos, a continuación, a través de la Tabla 3 , se presenta una matriz de correlaciones de las variables analizadas en este trabajo, donde se puede visualizar de una manera sencilla, las variables que presentan una mayor relación entre ellas. Se han resaltado en negrita los cinco resultados más elevados. 
Tabla 3. Matriz de correlaciones entre variables

\begin{tabular}{|c|c|c|c|c|c|c|c|c|c|}
\hline & fila & posición & centro & mod.bach & acceso & nota.acceso & sexo & actitud & examen \\
\hline fila & & -0.028 & 0.131 & 0.078 & -0.004 & -0.154 & -0.496 & -0.590 & -0.273 \\
\hline posición & & & -0.035 & 0.023 & -0.038 & 0.294 & 0.342 & 0.094 & 0.078 \\
\hline centro & & & & -0.070 & -0.319 & -0.098 & -0.011 & -0.101 & 0.010 \\
\hline mod.bach & & & & & 0.218 & 0.061 & -0.206 & 0.036 & 0.115 \\
\hline acceso & & & & & & 0.115 & -0.089 & 0.074 & -0.073 \\
\hline nota.acceso & & & & & & & 0.235 & 0.470 & 0.584 \\
\hline sexo & & & & & & & & 0.246 & 0.089 \\
\hline actitud & & & & & & & & & 0.646 \\
\hline examen & & & & & & & & & \\
\hline
\end{tabular}

Fuente: Elaboración propia

Así, se podría destacar, por ejemplo, que el número de fila ocupado y el sexo presentan una correlación prácticamente del 50\% negativa; esto nos indicaría que las mujeres se suelen ubicar en filas relativamente delanteras; algo que también se ha podido examinar a través de la Figura 3. Asimismo, se puede observar que normalmente una ubicación en filas delanteras conlleva una mejor nota de actitud, con una correlación del -0.59; algo que también se señalaba previamente. A su vez, una mejor actitud implica generalmente una mayor nota de examen, presentando una correlación del 0.64. Finalmente, cabe destacar que una mayor nota de acceso suele tener influencia positiva tanto sobre el examen final (0.47) como sobre la nota relativa a la actitud (0.58); algo que, según se ha observado previamente, únicamente ocurría en el caso particular de los star-students, los cuales contaban con 0.9 puntos más de media en su nota de acceso frente al resto de estudiantes, lo cual, según esta matriz de correlaciones influiría en su nota de actitud y examen final. De hecho, tal y como se indicaba con anterioridad, este tipo de estudiantes obtuvieron 1.9 puntos y 2.4 puntos más en actitud y examen respectivamente.

Para finalizar, y con el propósito de mejorar la robustez de los resultados presentados en este trabajo, se llevaron a cabo distintas regresiones lineales múltiples sobre la nota de examen final y la nota de actitud en función del resto de variables. Con ello, se pretendía identificar, aquellas variables con un mayor peso sobre estas dos calificaciones. Tras aplicar técnicas de regresión lineal múltiples "paso a paso" a través del software R, los modelos obtenidos fueron los siguientes:

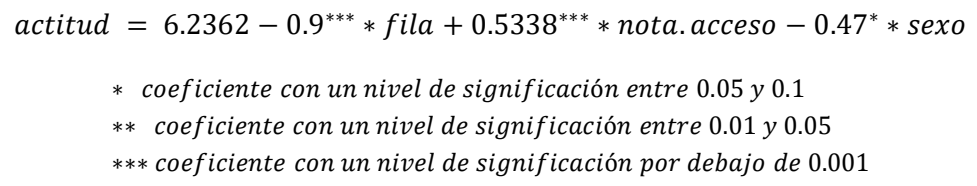

Este primer modelo cuenta con un p-valor de $3.3 * 10^{-13}$, mientras que su valor de $\mathrm{R}^{2}$ es de 0.51 . Asimismo, como puede observarse, su coeficiente más significativo es la fila, con un nivel de significación por debajo de $10^{-6}$. Esto implicaría que por cada fila que el estudiante retrasara su posición en el aula, su nota de actitud disminuiría en casi un punto. Estos resultados no harían más que afianzar los obtenidos previamente 
a nivel descriptivo, donde se señalaba una diferencia de 2.4 puntos (es decir, cercana a los 2.7 puntos indicados por este modelo) en el caso de sentarse en la última fila frente a realizarlo en la primera; es decir, tres filas más atrás.

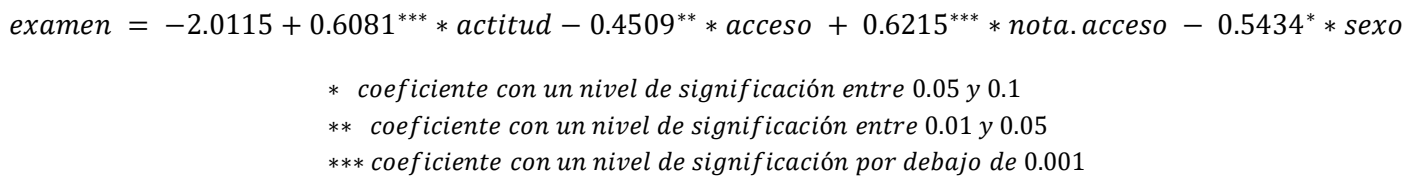

El segundo modelo cuenta con un p-valor de $2.497 * 10^{-14}$, mientras que su valor de $\mathrm{R}^{2}$ es de 0.56 . Del mismo modo, sus coeficientes más significativos son la actitud y la nota de acceso, con un nivel de significación por debajo de $10^{-5}$. Es importante recordar que, a través del primer modelo, podíamos observar como la actitud estaba altamente influenciada por la fila que ocupaba el estudiante; luego implícitamente, la fila ocupada también influiría a la nota del examen. De esta manera, por cada punto más de actitud en clase, la nota del examen final aumentaría en 0.6 puntos aproximadamente. Estos resultados irían en línea de los obtenidos a nivel descriptivo, donde se señalaba una diferencia de 1.3 puntos en la nota del examen en el caso de sentarse en la última fila frente a realizarlo en la primera; es decir, tres filas más atrás. Asimismo, la nota de acceso también tendría un peso muy elevado en este modelo, señalando que por cada punto más en esta nota, el examen final se vería incrementado en 0.62 puntos. Algo que, a nivel descriptivo, veíamos que también ocurría, aunque en esta ocasión, únicamente en el caso particular de los star-students; los cuales, obtenían 0.9 puntos más que el conjunto global de estudiantes en su nota de acceso, así como 1.9 puntos y 2.4 puntos más en actitud y examen. Además, cabe mencionar para este modelo que el coeficiente relativo a la modalidad de acceso, aunque con menos peso, nos indicaría que los estudiantes que acceden por vías internacionales u otras vías, obtendrían al final una peor calificación en el examen final.

Para finalizar, en ambos modelos la variable sexo aparece con signo negativo (aunque con un nivel de significación bastante bajo), lo cual significaría que el mero hecho de ser mujer generaría peores resultados. Concretamente -0.47 puntos en el caso de la actitud y de -0.54 en el caso del examen final. Sin embargo, esto iría en contra de los resultados obtenidos a nivel descriptivo, donde se señalaba incluso que las mujeres podrían ser potencialmente las causantes del incremento de nota obtenido en determinadas zonas del aula. En concreto, se argumentaba que estas presentaban 0.8 puntos más en actitud ( 7.7 frente a 6.9) y 0.4 puntos más en examen final (5.6 frente a 5.2) que los hombres. Una posible interpretación del modelo sería el mero hecho de que las propias mujeres obtuvieran mejores calificaciones en actitud que los hombres (en concreto, 0.8 puntos más de media). De esta manera, esta circunstancia podría ser la causante de que su signo fuera negativo; pues su resultado, se vería compensado con la nota de actitud obtenida al ser mujer, dando como output final, un balance positivo.

\section{Conclusiones}

En la literatura, diversos autores han analizado cómo la ubicación de los estudiantes en el aula afecta a su rendimiento académico y motivación durante las clases. Su explicación se ha basado en dos corrientes de pensamiento totalmente opuestas. La primera, se centra en argumentar que los estudiantes más inteligentes, participativos y con mayor motivación por sacar buenas notas preferirían los primeros puestos. Es decir, determinando que el lugar elegido por estos estaría muy correlacionado con sus características internas. Por otro lado, la segunda línea de investigación considera a la localización en el aula como el elemento más 
importante a la hora de generar mejores niveles de participación y resultados. En este sentido, el 'efecto fila' y la mayor atención recibida por parte de los profesores, tanto implícita como explícita, afectaría a su motivación, incidiendo en su esfuerzo por obtener unos mejores resultados académicos.

La presente contribución se enmarca dentro de esta área de investigación. Para ello, se llevó a cabo un estudio de las calificaciones de los estudiantes del primer curso académico del Grado en ADE para Emprendedores del Centro Universitario EDEM de Valencia. Concretamente, este análisis desarrollado a lo largo de tres asignaturas de distintos caracteres ha permitido hallar una relación entre la ubicación en el aula de los alumnos y sus calificaciones obtenidas. Así, y bajo la configuración específica de las aulas de EDEM (con un número reducido de estudiantes y organizadas en forma de hemiciclo), se constata que los estudiantes que obtienen mejores notas, suelen ubicarse en las primeras filas, tal y como establece la literatura en la actualidad (es decir, a través del segundo enfoque de investigación o 'efecto fila'). Sin embargo, este efecto viene acompañado también por un sesgo en las notas obtenidas por los estudiantes según su posición. En concreto, los resultados muestran cómo los alumnos ubicados en el centro y en la derecha del aula presentan mejores calificaciones que el resto. Algo que, en el caso concreto de los estudiantes ubicados a la derecha de la misma, no ha sido evidenciado previamente por la literatura. Como principal hipótesis se establece la ubicación del atril en el aula, el cual, a su vez podría afectar a la atención prestada por el profesor durante sus explicaciones. No obstante, a través de este trabajo, se insta a futuras investigaciones a ahondar sobre sus posibles causas, como pudiera ser la presencia de ventanales con un exceso de luz en ese lado del aula, la falta o ausencia de espacio en la pizarra debido a la presencia del propio atril, etc., que pudiera hacer que el docente involuntariamente prestara una menor atención a los estudiantes ubicados en esta zona.

Por otro lado, el presente trabajo busca unificar las dos teorías previamente expuestas y aparentemente enfrentadas, bajo la inclusión del concepto del star-student (o estudiante estrella). Su análisis, basado en la ubicación de los mismos en el aula, demuestra que este tipo de estudiante suele preferir sentarse en las primeras filas, apoyando de esta forma la primera línea de investigación basada en las características internas del alumno (o 'efecto individuo'). Asimismo, al incluir la variable género en el estudio, se consigue revelar que su efecto es prácticamente insignificante dentro de dichos estudiantes excelentes. Es decir, el género no sería relevante entre los alumnos más inteligentes, pudiendo ser estos hombres o mujeres indistintamente. Sin embargo, para el conjunto global de los mismos sí sería relevante, generando calificaciones más elevadas en las mujeres respecto a los hombres; las cuales, curiosamente, se localizarían también en dichas primeras filas del aula.

A través de este trabajo se pretende usar esta información de cara a próximos semestres o cursos, tanto a nivel de asignaturas concretas como a nivel de centro, para poder conocer mejor la ubicación de determinados tipos de estudiantes en el aula. De esta manera, se podría ayudar al docente a considerar sus necesidades específicas o, incluso, modificar la localización de los mismos en el aula, con el fin de mejorar sus rendimientos académicos.

De una forma similar, estas conclusiones podrían ser útiles para los estudiantes, los cuales podrían utilizarlas individualmente o en grupo, para ser conscientes de su ubicación dentro del aula y prever los posibles efectos que esta tendría sobre sus resultados. De esta manera, este mejor conocimiento de la realidad, podría afectar a su automotivación a la hora de incrementar su atención en el aula o, incluso, hacerles cambiar su sitting en aras de mejorar sus calificaciones. 


\section{Limitaciones y futuras lineas de investigación}

Como posibles limitaciones al estudio, cabe considerar en primer lugar que, a pesar de analizar a los mismos estudiantes a lo largo de tres asignaturas, las cuales representan adecuadamente las tres principales áreas de conocimiento del grado (entorno interno/emprendedor, entorno externo/empresarial y entorno técnico/de implementación), dicho estudio se desarrolló únicamente durante un semestre. Esto abre una gran oportunidad para continuar con este tipo de análisis en un futuro, pudiendo analizar el conjunto de asignaturas cursadas durante un mismo curso académico, o incluso, a lo largo de todo el grado. Con ello, se obtendrían datos mucho más precisos y se podrían dirimir soluciones más concretas.

En segundo lugar, es importante tener en cuenta que a pesar de la relevancia y novedad de la inclusión de la variable género en este estudio, cabría determinar que las mujeres únicamente representan el $27 \%$ de la muestra. Es por ello que, desde este trabajo, se insta a profundizar en este ámbito; ya sea a través de poblaciones más homogéneas o con un mayor número de mujeres en las mismas.

En tercer lugar, podría argumentarse que el aprendizaje del alumnado podría ser también subjetivo, y que ellos mismos, podrían establecer su grado de satisfacción con los contenidos y conocimientos adquiridos por parte de ese profesor y asignatura. Es lo que Kalinowski y Toper (2007) denominan como 'percepción del alumno' y se podría conseguir simplemente analizando dos de las preguntas que los estudiantes de EDEM ya contestan a través de sus evaluaciones periódicas: "Estoy satisfecho con el profesor de esta asignatura" y "Estoy satisfecho con la docencia en esta asignatura". Sin embargo, para un primer nivel de análisis, en este trabajo se ha considerado más óptimo centrarse únicamente en variables objetivas. Esto, no obstante, no impediría ampliar (y enriquecer) en un futuro el horizonte de esta investigación, realizando comparativas entre ambos tipos de evaluaciones.

Finalmente, y aunque no se perciba como una limitación específica, es importante resaltar que este estudio no debería considerarse como un alegato irrefutable al cambio de ubicación de aquellos alumnos que peores calificaciones obtienen. De esta forma, además de tener en cuenta el efecto de la configuración de la clase, tal y como argumentan numerosos investigadores, habría que considerar también aspectos más internos de los estudiantes, como pudieran ser características emocionales y psicológicas de los mismos (Marshall y Lonsoczy, 2010; Smith et al., 2018). Así, un ejemplo de ello podría ser el considerar el efecto que podría tener sobreexponer a un estudiante tímido (o invisibilizar a uno motivado) en su proceso de aprendizaje y desarrollo cognitivo. Lo mismo podría suceder al separar a determinados grupos de alumnos pues, aunque parezca una trivialidad, numerosos estudios demuestran que los estudiantes que se sientan juntos por afinidad obtienen mejores calificaciones (además, siendo estas, muy parecidas entre las mismas).

\section{Referencias}

Benedict, M. E., y Hoag, J. (2004). Seating location in large lectures: Are seating preferences or location related to course performance?. The Journal of Economic Education, 35(3), 215-231.

Burda, J. M., y Brooks, C. I. (1996). College classroom seating position and changes in achievement motivation over a semester. Psychological Reports, 78(1), 331-336.

Holliman, W. B., y Anderson, H. N. (1986). Proximity and student density as ecological variables in a college classroom. Teaching of Psychology, 13(4), 200-203.

Kalinowski, S., y Toper, M. L. (2007). The Effect of Seat Location on Exam Grades and Student Perceptions in an Introductory Biology Class. Journal of College Science Teaching, 36(4). 
Marshall, P. D., y Losonczy-Marshall, M. (2010). Classroom ecology: relations between seating location, performance, and attendance. Psychological reports, 107(2), 567-577.

Smith, D. P., Hoare, A., y Lacey, M. M. (2018). Who goes where? The importance of peer groups on attainment and the student use of the lecture theatre teaching space. FEBS open bio, 8(9), 1368-1378.

Stires, L. (1980). Classroom seating location, student grades, and attitudes: Environment or self-selection?. Environment and behavior, 12(2), 241-254.

Worthington, D. L., y Levasseur, D. G. (2015). To provide or not to provide course PowerPoint slides? The impact of instructor-provided slides upon student attendance and performance. Computers \& Education, $85,14-22$. 